\author{
Yu-Tzu Tsao \\ Wei-Liang Chen \\ Jui-Chang Chen
}

\section{Pathergy phenomenon: huge pyoderma gangrenosum following tube thoracostomy}

Received: 30 July 2014

Accepted: 1 August 2014

Published online: 15 August 2014

(C) Springer-Verlag Berlin Heidelberg and ESICM 2014

\section{Y.-T. Tsao $(\bowtie) \cdot$ J.-C. Chen}

Department of Critical Care Medicine, Taoyuan General Hospital, Ministry of Health and Welfare, No. 1492, Chung-Shan Road,

Taoyuan, Taiwan

e-mail: tsaoyutzu@gmail.com

Tel.: 886-3-369-9721

\section{W.-L. Chen}

Department of Community Medicine, Tri-Service General Hospital, Taipei, Taiwan

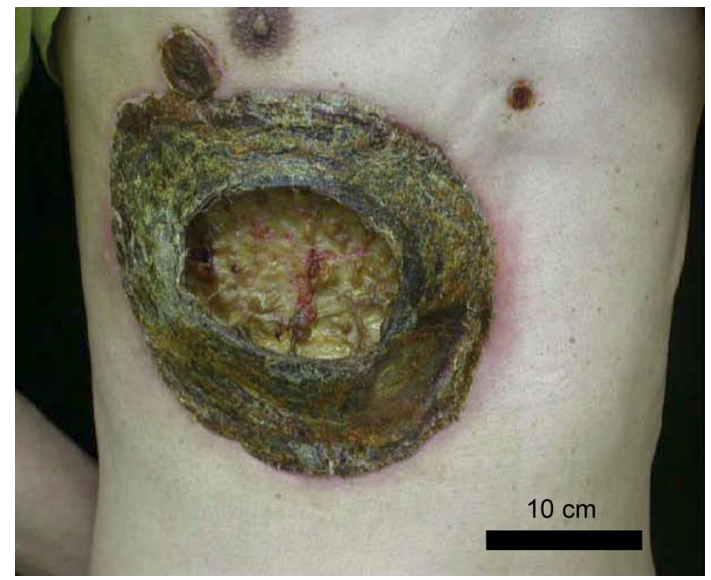

Fig. 1 A giant cutaneous ulcer occupying the entire right chest wall showing a large purulent base covered by necrotic eschars and a surrounding erythematous halo, characteristic of pyoderma gangrenosum

painful, irregular, boggy, red-purple ulcers with an undermined purple-to-red margin and a halo of surrounding erythema. It can develop at sites of skin trauma, a cutaneous phenomenon known as pathergy. The etiology is unclear and there is no confirmative laboratory test or pathognomonic histological feature. The diagnosis is one of exclusion. Systemic corticosteroids are considered the most predictable and effective therapy. Surgical treatment may worsen skin lesions and should be avoided. Clinicians must maintain a heightened awareness of this distinct manifestation.

Conflicts of interest None declared. 